Check for updates

Cite this: RSC Adv., 2017, 7, 56721

\section{First-principles analysis of the stability of water on oxidised and reduced $\mathrm{CuO}(111)$ surfaces}

\begin{abstract}
Marco Fronzi (iD *ab and Michael Nolan (iD ${ }^{\mathrm{b}}$
We use first-principles density functional theory calculations including the Hubbard $+U$ correction (PBE + $U)$ on $\mathrm{Cu}-3 \mathrm{~d}$ states to investigate the interaction of water with a $\mathrm{CuO}(111)$ surface. We compute adsorption energies and the stability of different water coverages, with a particular focus on the interaction of water with oxygen vacancy sites, and how vacancy stabilization occurs. We study the energetics, geometry and electronic structure of relevant configurations, finding that there are only small changes to the local geometry around the water adsorption site(s) and the electronic properties. The inclusion of van der Waals interactions has no significant impact on the stability of water on $\mathrm{CuO}(111)$. We extend the analysis to include realistic environmental conditions within the ab initio atomistic thermodynamics framework, which allows us to assess the stability of the water/copper-oxide system as a function of ambient conditions, and focus on three important surface processes: water adsorption/desorption on the stoichiometric surface, conditions for dissociation, and oxygen vacancy stabilization.
\end{abstract}

Received 27th October 2017 Accepted 30th November 2017 DOI: 10.1039/c7ra11854f

rsc.li/rsc-advances
LDA or GGA functionals, which give a metallic system. Corrections such as self-interaction correction, DFT $+U$ or hybrid DFT can open up the band gap and recover the anti-ferromagnetic structure, to give a consistent description of the properties of CuO. ${ }^{9-16}$

For the possible exploitation of $\mathrm{CuO}$ in solar to chemical energy conversion, generically termed artificial photosynthesis, we also have to consider, in addition to the properties of $\mathrm{CuO}$, the fact that water will most likely be present and water can interact with metal oxide surfaces to modify them compared to the pristine surfaces. Water can be present either in the atmosphere or within the solution in which the reaction takes place. Thus, from one perspective, water may actively participate in the chemical reactions, contributing by donating either hydrogen or oxygen atoms, or from another perspective the water present can dissociate to form surface hydroxyl groups, which can then alter the properties of the catalyst.

Unlike other metal oxide surfaces such as (110) rutile and (101) anatase $\mathrm{TiO}_{2}$ or $\mathrm{CeO}_{2}(111),{ }^{17-19,35,36}$ little is known about the interaction of water with $\mathrm{CuO}$ surfaces. An attempt to study surface-water interactions has been presented by Zhang et al. ${ }^{\mathbf{2 0}}$ They investigated the interaction of a single water molecule with perfect and defective $\mathrm{CuO}(111)$ surfaces using density functional theory within the GGA approximation, using the Perdew and Wang functional (PW91) for the exchange-correlation term where copper atoms were kept frozen and substituted by a DFT semi-core pseudopotential and other atoms are treated with an all-electron basis set. However, they neglected to include any consistent description of localized electron in the $\mathrm{Cu}-3 \mathrm{~d}$ shell. The authors obtained adsorption energies and dissociation barriers, and concluded that the
${ }^{a}$ International Research Centre for Renewable Energy, State Key Laboratory of Multiphase Flow in Power Engineering, Xi'an Jiaotong University, Xi'an 710049, Shaanxi, China.E-mail: marco.fronzi@mail.xjtu.edu.cn

${ }^{b}$ Tyndall National Institute, University College Cork, Lee Maltings, Cork, Ireland 
oxygen vacancy can exhibit a strong catalytic activity towards $\mathrm{H}_{2} \mathrm{O}$ dissociation. However, by not including any correction for the electron self-interaction, this can cause issues in describing hydrogen and hydroxyl interactions at and vacancies on the $\mathrm{CuO}(111)$ surface. In another study of $\mathrm{CuO}(111)$, it was shown by Maimaiti et al. that $\mathrm{CuO}$ shows some activity with respect to water formation as a result of the interaction of hydrogen with $\mathrm{CuO}^{21}$ In particular the most stable $\mathrm{CuO}(111)$ surface was investigated and the authors demonstrated the formation of water after $\mathrm{H}_{2}$ adsorption, with surface oxygen atoms being removed. However, the adsorption and dissociation of $\mathrm{H}_{2} \mathrm{O}$ on the $\mathrm{CuO}(111)$ surface under realistic environmental conditions and at different coverages, within a consistent theoretical approach, is not at all understood and therefore a detailed analysis of the interaction of water with the $\mathrm{CuO}(111)$ surface under realistic catalytic conditions is still required and forms the subject of this paper.

We present a significant advance on these previous investigations, to give new insights into the stability of water at different coverages under different conditions on $\mathrm{CuO}(111)$. We analyse the properties of water adsorption on the clean and defective (111) surface at different coverages using first principles density functional theory, within the Generalized Gradient Approximation (GGA) using the Perdew-Burke-Ernzerhof (PBE) functional for the exchange-correlation term. To correct the unphysical electron self-interaction that is present in the localized $\mathrm{Cu}-3 \mathrm{~d}$ shell, which is a fundamental problem in strongly correlated systems, we apply the Hubbard $+U$ correction, giving so-called $\mathrm{PBE}+U$. PBE $+U$ has been applied to describe the localized $\mathrm{Cu}-3 \mathrm{~d}$ states in bulk $\mathrm{CuO}$ and in $\mathrm{Cu}$ doping of metal oxides, and has been successful in correctly describing many properties of $\mathrm{Cu}^{\mathbf{8}, 21,22}$ We further extend the analysis of the water-CuO interaction to treat more realistic environmental conditions, using our PBE $+U$ energies within the framework of the $a b$ initio atomistic thermodynamics formalism to model our system when in contact with a humid atmosphere consisting of oxygen and water vapour. ${ }^{23,24}$ Taken together, these results will give new insights into the interaction of $\mathrm{CuO}(111)$ and water, giving an understanding of the potential reactivity of $\mathrm{CuO}$ in a water environment.

\section{Calculation methods}

The calculations presented in this work are performed using a density functional theory approach, within the Generalized Gradient Approximation (GGA) using the Perdew-Burke-Ernzerhof (PBE) functional for the exchange-correlation term. ${ }^{25} \mathrm{We}$ use periodic boundary conditions with a plane wave basis set as implemented in the VASP code. ${ }^{26}$ The cut-off energy for the valence electrons has been set at $400 \mathrm{eV}$, and the core-valence interaction is described by the projected augmented wave approach, with 11 valence electrons for $\mathrm{Cu}, 6$ for $\mathrm{O}$ and 1 for $\mathrm{H}^{27}$ $\Gamma$ point calculations have been used for a $(2 \times 6)$ super-cell expansion of the $\mathrm{CuO}(111)$ surface, which consists of 24 atomic layers, with a vacuum gap of $20 \AA$. The convergence criteria for the energy and forces are $10^{-5} \mathrm{eV}$ and $10^{-5} \mathrm{eV} \AA^{-1}$, respectively.
In order to correctly describe the partially occupied states of $\mathrm{Cu}-3 \mathrm{~d}$ and $\mathrm{O}-2 \mathrm{p}$, the use of the Hubbard correction becomes crucial. The partially occupied states are described with $U(\mathrm{Cu}-$ $3 \mathrm{~d})=4.0 \mathrm{eV}$ and $U(\mathrm{O}-2 \mathrm{p})=5.5 \mathrm{eV}$. These are in line with previous works. ${ }^{21}$ For some specific cases, a value of $7.0 \mathrm{eV}$ has been also employed for the $\mathrm{Cu}-3 \mathrm{~d}$ state, in order to test trends in the key quantities as a function of the magnitude of the Hubbard $+U$ term. In previous studies, we have found that the $+U$ correction is required to describe $\mathrm{O}-2 \mathrm{p}$ hole states that result after valence band hole formation; this is relevant for our ongoing work in studying the excited states of metal oxides. ${ }^{28,29}$ This $+U$ correction on oxygen can also improve the binding energy of $\mathrm{O}_{2}$ which is important for vacancy formation. To estimate the contribution of van der Waals ( $\mathrm{vdW}$ ) interactions arising from the interaction of electric dipole moments, we repeated the calculations including a correction developed by Grimme (DFT-D3), and report the effect on the geometries and energetics. $^{30}$ Using this set up we found that the antiferromagnetic configuration is the most stable for bulk $\mathrm{CuO}$, and our computed lattice constants are $a=4.683 \AA$, $b=3.43 \AA$, $c=5.138 \AA$, and $\beta=99.2^{\circ}$.

\section{Results}

\subsection{Energetics of different water adsorption coverages on $\mathrm{CuO}(111)$}

In this section we analyse the geometry and energetics of the interaction between water and $\mathrm{CuO}(111)$ surfaces, focusing on the adsorption of water at different coverages. The geometry of water at each coverage has been determined by the structural optimization of different initial water adsorption configurations. The adsorption energies are calculated per adsorbed water molecule as follows:

$$
E=\frac{1}{n} E_{\mathrm{CuO}+\mathrm{H}_{2} \mathrm{O}}-\left[E_{\mathrm{CuO}}+n E_{\mathrm{H}_{2} \mathrm{O}}\right]
$$

where $E_{\mathrm{CuO}+\mathrm{H}_{2} \mathrm{O}}, E_{\mathrm{CuO}}$ and $E_{\mathrm{H}_{2} \mathrm{O}}$ are the energies of the CuO-water surface slab, the $\mathrm{CuO}(111)$ surface slab and free gas phase water, while $n$ is the number of water molecules adsorbed per $\mathrm{CuO}(111)$ super-cell. The $\mathrm{CuO}(111)$ surface forms a lattice consisting of four-coordinated copper atoms in the surface and subsurface regions and these are denoted $\mathrm{Cu}_{\text {surf1 }}$ and $\mathrm{Cu}_{\text {surf2 }}$, respectively (see Fig. 1). The $\mathrm{Cu}_{\text {surf1 }}$ and $\mathrm{Cu}_{\text {surf2 }}$ atoms take a 4coordinate square planar environment, with the former binding to surface and subsurface oxygen species. There are threecoordinated oxygen atoms located in the surface and subsurface layers, denoted as $\mathrm{O}_{\text {surf }}$ and $\mathrm{O}_{\text {sub }}$, respectively.

In a $(2 \times 6)$ expansion of the $\mathrm{CuO}$ super-cell, there are $24 \mathrm{Cu}$ atoms that represent potentially favourable water adsorption sites and when $1,3,6,8$ or 12 water molecules adsorb on the CuO surface, the corresponding water coverages are 1/24, 1/8, 1 / 4, $1 / 3$ and $1 / 2$ monolayer (ML). Tables 1 and 2 report the computed water adsorption energies calculated for different water coverages and for different adsorption modes, within our $\mathrm{PBE}+U$ computational set-up. Table 1 also reports a direct comparison with the adsorption energies for a single water molecule calculated by Zhang et al. in ref. 20. A single water 


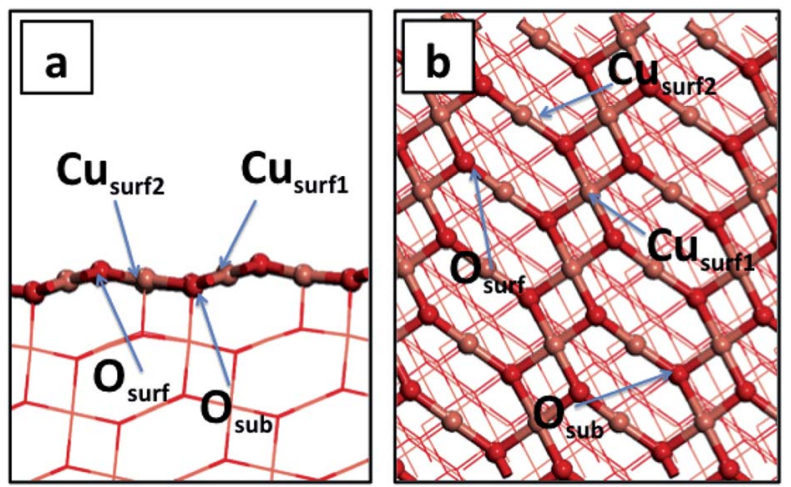

Fig. 1 Atomic structure of a clean $\mathrm{CuO}(111)$ surface, highlighting the different types of $\mathrm{Cu}$ and oxygen atom at the surface. Light red and dark red spheres represent copper and oxygen atoms, respectively.

Table 1 Adsorption energies of water at the $\mathrm{CuO}(111)$ surface at 1/24 $M L$ coverage. Energies are referenced to gas phase water and the bare $\mathrm{CuO}(111)$ surface

\begin{tabular}{lll}
\hline Coverage & Ads. $E(\mathrm{eV})$ & Ads. $E^{a}(\mathrm{eV})$ \\
\hline $1 / 24 \mathrm{ML}\left(\mathrm{Cu}_{\text {surf1 }}\right)$ & -0.29 & -0.40 \\
$1 / 24 \mathrm{ML}\left(\mathrm{Cu}_{\text {surf2 }}\right)$ & -0.32 & -0.90 \\
$1 / 24 \mathrm{ML}\left(\right.$ dissociated at $\left.\mathrm{Cu}_{\text {surf2 }}\right)$ & -0.35 & - \\
${ }^{a}$ Zhang et al. ref. 20. & &
\end{tabular}

Table 2 Adsorption energies of water (per molecule) at the $\mathrm{CuO}(111)$ surface at 1/8,1/4,1/3 and 1/2 water coverage for different adsorption configurations at $\mathrm{Cu}$ sites with different $\mathrm{Cu}_{\text {surf1 }} / \mathrm{Cu}_{\text {surf2 }}$ ratios. Here, dissociative means that, for the indicated coverage, one water molecule dissociates into a hydrogen atom and hydroxyl group. Energies are referenced to gas phase water and the bare CuO(111) surface
Coverage

1/8 molecular (Fig. 3(a))

1/8 molecular (Fig. 3(b))

1/8 molecular (Fig. 3(c))

1/8 dissociative (Fig. $3(\mathrm{~d})$ )

1/4 molecular (Fig. 4(a))

1/4 molecular (Fig. 4(b))

$1 / 4$ molecular (Fig. 4(c))

1/4 dissociative (Fig. $4(\mathrm{~d})$ )

1/4 dissociative (Fig. $4(\mathrm{e})$ )

$1 / 3$ molecular (Fig. 5(a))

1/2 molecular (Fig. 5(b))
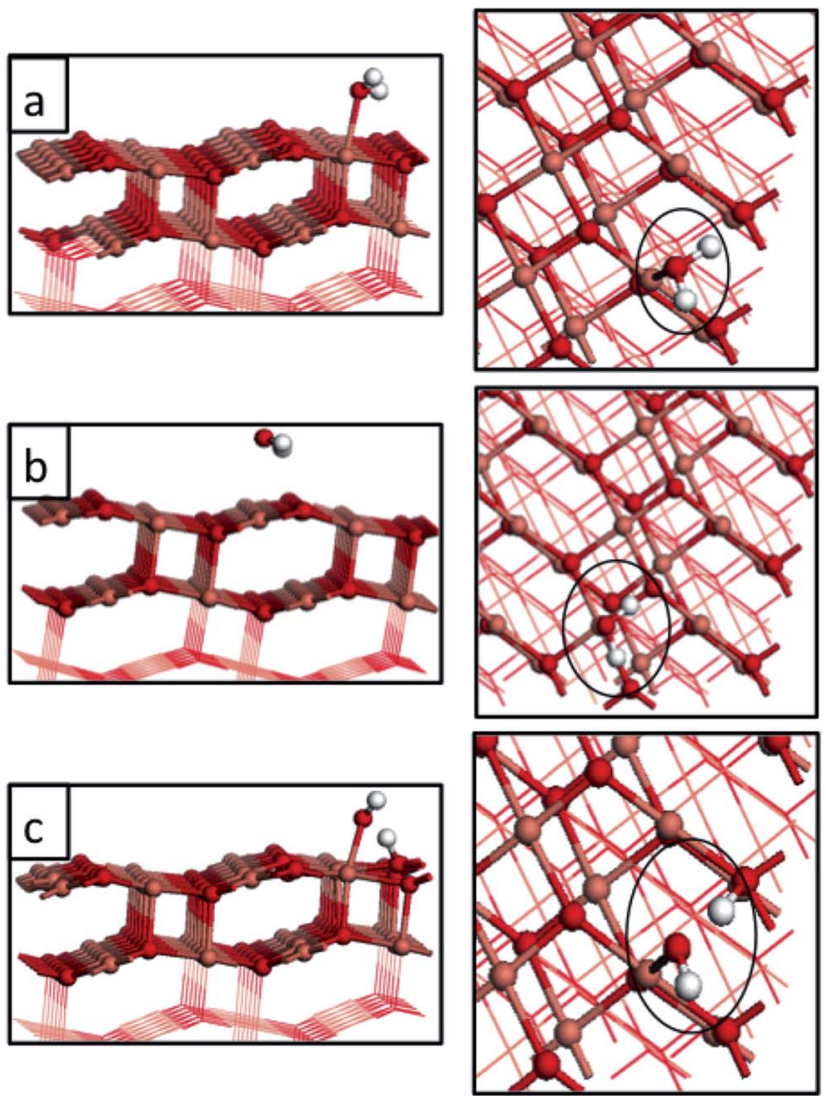

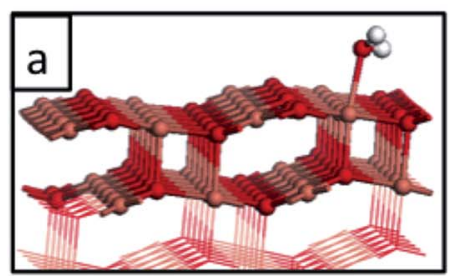

Fig. 2 Atomic structure of water adsorbed on the $\mathrm{CuO}(111)$ surface at coverages of 1/24 ML. Panels (a) and (b) represent molecular adsorption at two different $\mathrm{Cu}$ sites, namely $\mathrm{Cu}_{\text {surf2 }}$ and $\mathrm{Cu}_{\text {surf1 }}$ (see Fig. 1). Panel (c) represents the dissociative adsorption of water. Light red, dark red and white spheres represent copper, oxygen and hydrogen atoms, respectively.

multiple adsorbed water molecules, we have to consider adsorption configurations that take into account water adsorption at the $\mathrm{Cu}_{\text {surf } 1}$ and $\mathrm{Cu}_{\text {surf2 }}$ sites that were indicated in Fig. 1.

We find a significant effect of the computational methodology on the magnitude of the adsorption energy of a single water molecule on the $\mathrm{CuO}(111)$ surface. Zhang et al. calculate, using plain DFT, adsorption energies of a single water molecule to be $-0.90 \mathrm{eV}$ and $-0.41 \mathrm{eV}$ for adsorption at the $\mathrm{Cu}_{\text {surf2 }}$ and $\mathrm{Cu}_{\text {surf1 }}$, respectively, indicating a clear preference for the $\mathrm{Cu}_{\text {surf2 }}$ site. In contrast our water adsorption energies for 1/24 ML coverage at the same $\mathrm{Cu}$ sites are essentially iso-energetic using the $+U$ correction to the $\mathrm{Cu}-3 \mathrm{~d}$ states. This comparison indicates a very strong dependence on the description of the $\mathrm{Cu}^{2+}$ oxidation state, when going from $U(\mathrm{Cu})=0 \mathrm{eV}$ (incorrect description of $\mathrm{Cu}^{2+}$ ) to $U(\mathrm{Cu})=4.0 \mathrm{eV}$ (consistent description of $\left.\mathrm{Cu}^{2+}\right)$. Not only are the magnitudes of the adsorption energies reduced with the inclusion of the Hubbard $+U$ term, but the stabilities of the two $\mathrm{Cu}$ adsorption sites are now very similar. To further examine the effect of the magnitude of the $+U$ correction on the adsorption energies, we recalculated the 1/24 ML water adsorption energies with $U(\mathrm{Cu})=7.0 \mathrm{eV}$ and this 
changes the adsorption energy by only $0.02 \mathrm{eV}$, compared to the result determined with $U(\mathrm{Cu})=4.0 \mathrm{eV}$. This result indicates that the description of electron localization in $\mathrm{CuO}$ can play a crucial role in molecular adsorption. Its effect is to strongly modify the adsorption energy once $U$ is large enough to consistently describe the $\mathrm{Cu}^{2+}$ oxidation state. Further increases in the magnitude of $U$ lead to insignificant changes.

As we increase the water coverage, there are multiple adsorption modes to consider and these are shown for coverages of 1/8, 1/4, 1/3 and 1/2 ML of water in Fig. 3,4 and 5, and reported in Table 2. We have explored adsorption of water at exclusively $\mathrm{Cu}_{\text {surf2 }}$ or $\mathrm{Cu}_{\text {surf1 }}$ sites and a mix of $\mathrm{Cu}_{\text {surf } 2} / \mathrm{Cu}_{\text {surf1 }}$ sites and we find that the adsorption modes that maximise the number of $\mathrm{Cu}_{\text {surf2 }}$ sites involved are the most favourable for these coverages. As coverage increases, we observe a significant change in the water adsorption energies, which increases from $-0.32 \mathrm{eV}$ per water molecule at $1 / 24 \mathrm{ML}$ coverage to $-0.61 \mathrm{eV}$ per water molecule at high coverages of $1 / 2$ ML. A possible origin of the enhanced stability of water on $\mathrm{CuO}(111)$ with higher coverage is the increase in water-water interactions that
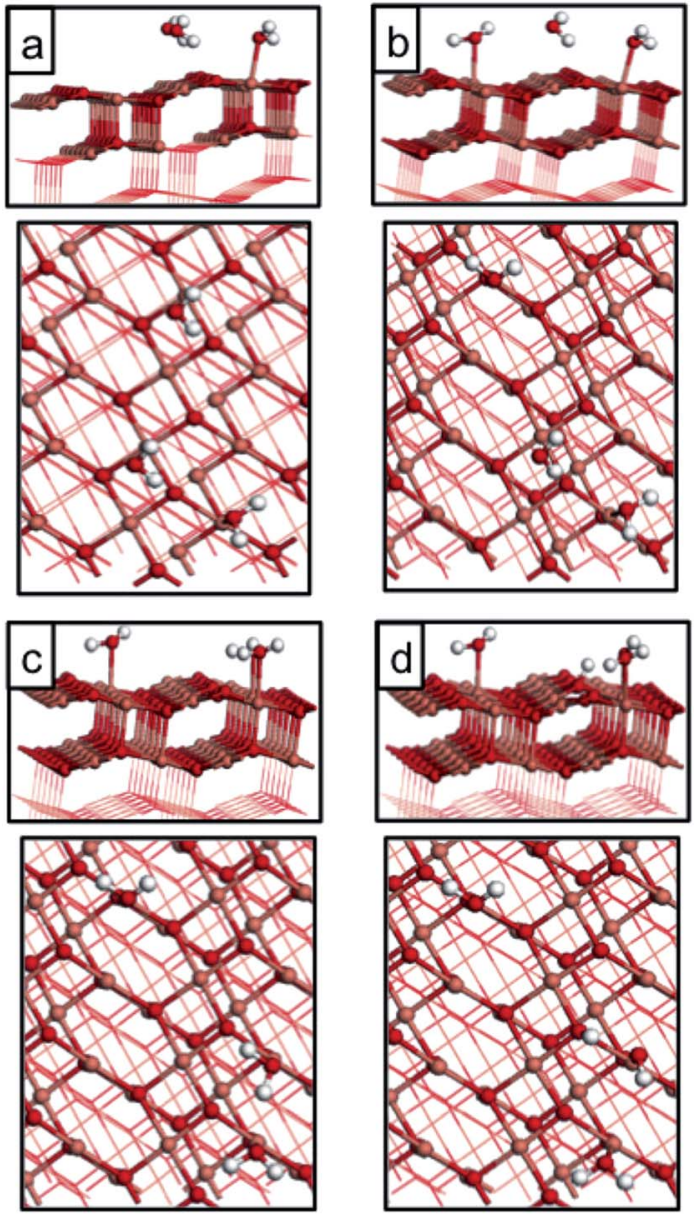

Fig. 3 Atomic structure of water adsorbed on the $\mathrm{CuO}(111)$ surface at coverages of $1 / 8 \mathrm{ML}$. Panels (a)-(c) represent different molecular adsorption configurations at both $\mathrm{Cu}_{\text {surf1 }}$ and $\mathrm{Cu}_{\text {surf2, }}$ and panel (d) represents a dissociated adsorption mode upon adsorption at $\mathrm{Cu}_{\text {surf2 }}$. Light red, dark red and white spheres represent copper, oxygen and hydrogen atoms, respectively.
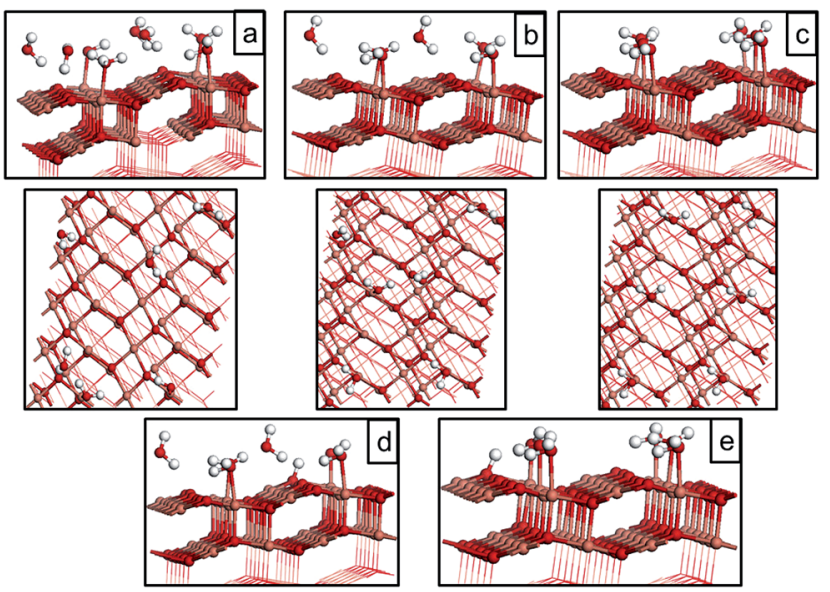

Fig. 4 Atomic structure of water adsorbed on the $\mathrm{CuO}(111)$ surface at coverages of 1/4 ML. Panels (a)-(c) represent different adsorption configurations of molecular water at this coverage at both $\mathrm{Cu}_{\text {surf } 1}$ and $\mathrm{Cu}_{\text {surf2 }}$ for (a) and (b), and only $\mathrm{Cu}_{\text {surf2 }}$ for (c). Panels (d) and (e) represent different dissociative adsorption configurations at this coverage upon adsorption at both $\mathrm{Cu}_{\text {surf1 }}$ and $\mathrm{Cu}_{\text {surf2 }}$ in panel (d), and $\mathrm{Cu}_{\text {surf2 }}$ in panel (e). Light red, dark red and white spheres represent copper, oxygen and hydrogen atoms, respectively.

could stabilize the high coverages, which in our system was calculated to be $0.1 \mathrm{eV}$ per water molecule at $1 / 2 \mathrm{ML}$ coverage, driven by the preference to adsorb water molecules along the $\mathrm{Cu}_{\text {surf2 }}$ sites which allows strong water-water interactions.

We repeated the calculations with the inclusion of the $\mathrm{vdW}$ functional at different coverages, and the computed adsorption energies show an increased stability of $\sim 0.11 \mathrm{eV}$ per water molecule at each coverage. For dissociation of a single water molecule, the adsorption energy increases by $0.18 \mathrm{eV}$, suggesting that the relative stability trend will not change with the inclusion of the vdW correction. Furthermore, water-water interactions are not significantly affected by non-local correction to DFT, and we calculated this contribution to be $0.02 \mathrm{eV}$
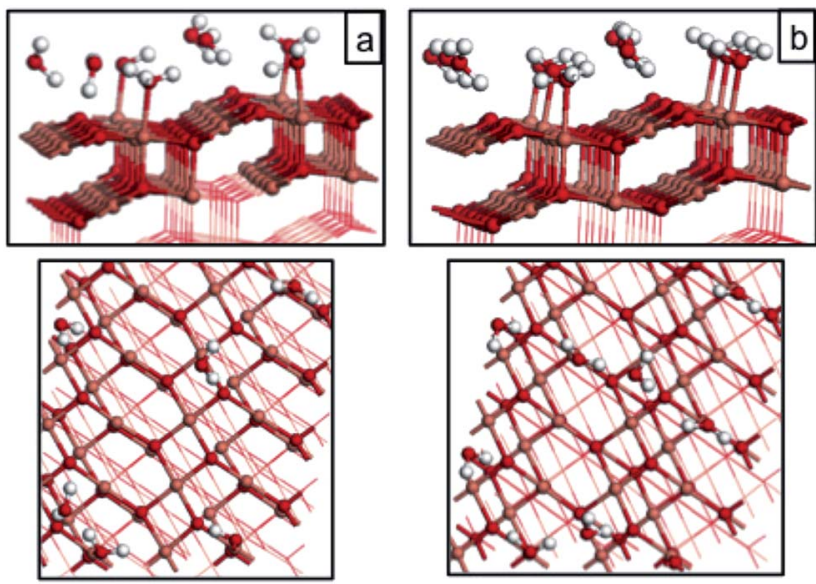

Fig. 5 Atomic structure of water adsorbed on the $\mathrm{CuO}(111)$ surface at coverages of (a) $1 / 3 \mathrm{ML}$ and (b) $1 / 2 \mathrm{ML}$ at both $\mathrm{Cu}_{\text {surf } 1}$ and $\mathrm{Cu}_{\text {surf2. }}$. Light red, dark red and white spheres represent copper, oxygen and hydrogen atoms, respectively. 
per water molecule in $1 / 2 \mathrm{ML}$ adsorption. This is consistent with the results obtained by Carrasco et al., who showed for water adsorption on $\mathrm{CeO}_{2}(111)$ that the relative stability, adsorption sites, and adsorption geometries of competing water adstructures rarely differ when comparing results obtained with semilocal functionals and non-local vdW density functionals. ${ }^{31}$

\subsection{Geometric and electronic properties of water adsorbed on $\mathrm{CuO}(111)$ at different coverages}

Table 3 reports, for each adsorption configuration at each coverage corresponding to the adsorption configuration energies reported in Tables 1 and 2, important atomic distances for molecular and dissociated water. The average distance between $\mathrm{Cu}$ and water oxygen is significantly different, with a value between 1.99-2.12 $\AA$ at $\mathrm{Cu}_{\text {surf1 }}$ and 2.77/3.11 $\AA$ at $\mathrm{Cu}_{\text {surf2 }}$. The trend shows an average short distance with increasing the coverage, however this effect is less evident at $\mathrm{Cu}_{\text {surf2 }}$, due to a weaker $\mathrm{Cu}-\mathrm{O}$ interaction. Also, when increasing the coverage, the $\mathrm{Cu}-\mathrm{OH}$ distance increases, although this effect is not as pronounced, and there is an elongation in the $\mathrm{O}-\mathrm{H}$ bond distance in water molecules.

The pristine $\mathrm{CuO}$ surface has six $\mathrm{Cu}-\mathrm{O}$ distances, as illustrated in Fig. 6. After water adsorption we examined the lattice distortions associated with the change in $\mathrm{Cu}-\mathrm{O}$ distance upon water adsorption. When a single water molecule adsorbs at the $\mathrm{Cu}_{\text {surf } 1}$ site, we find a change in $\mathrm{Cu}_{\text {surf } 1}-\mathrm{O}_{\text {surf }}$ and $\mathrm{Cu}_{\text {surf } 1}-\mathrm{O}_{\text {sub }}$ distances to $1.91 \AA$ and $1.99 \AA$, respectively, and $\mathrm{Cu}_{\text {surf2 }}-\mathrm{O}_{\text {surf }}$ distances to $1.93 \AA$ and $1.89 \AA$, while a second layer $\mathrm{O}$ atom shifts toward $\mathrm{Cu}_{\text {surf1 }}$ by $0.01 \AA$. On the other hand, when water adsorbs at the $\mathrm{Cu}_{\text {surf2 }}$ site, $\mathrm{Cu}_{\text {surf } 2}-\mathrm{O}$ distances will have a change between $0.02-0.06 \AA$, while the $\mathrm{Cu}_{\text {surf2 }}$ distance from the second layer oxygen is not significantly affected. We do not observe a significant deviation by this trend in the lattice distortion at higher water coverages. When the vdW correction is introduced, our calculated $\mathrm{Cu}-\mathrm{O}$ and $\mathrm{Cu}-\mathrm{H}$ distances are, in both the high and low water coverage, around $0.02 \AA$ and $0.03 \AA$, respectively, shorter than plain $\mathrm{PBE}+U$.

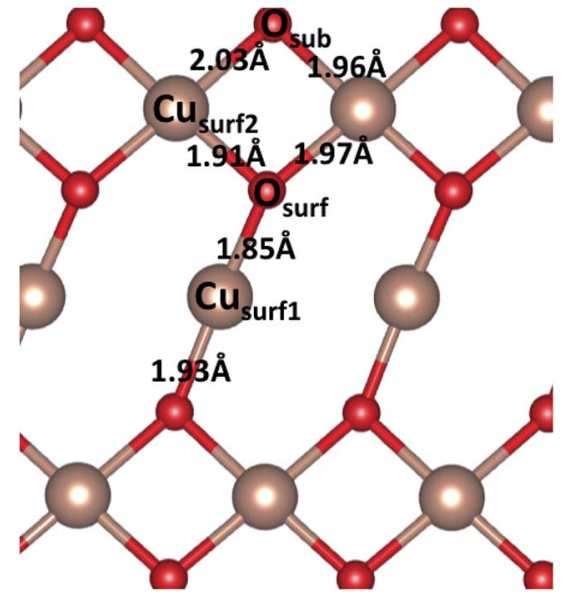

Fig. 6 Schematic representation of the $\mathrm{CuO}(111)$ pristine surface, indicating the two $\mathrm{Cu}$ and two $\mathrm{O}$ sites. The figure reports $\mathrm{Cu}-\mathrm{O}$ bond distances.

Turning now to the electronic properties of water covered $\mathrm{CuO}(111)$, we examine the computed Bader charges and spin magnetisations in bare $\mathrm{CuO}(111)$ and after water adsorption. In the bare $\mathrm{CuO}(111)$ surface, the computed Bader charges are 9.8 electrons, which are typical values of $\mathrm{Cu}^{2+}$ species in different systems. The computed spin magnetisations are $0.5 \mu_{\mathrm{B}}$ per atom, which are in reasonable agreement with the experimental magnetic moment of $0.68 \mu_{\mathrm{B}}$ and a significant improvement on the GGA results with no $+U$ correction, which are $0 \mu_{\mathrm{B}}{ }^{21}$ When we examine the Bader charges and spin magnetisations for the different water adsorption modes we find that these deviate very little from the pure $\mathrm{CuO}$ values. This suggests that the interaction of water with $\mathrm{CuO}$ surfaces induces little perturbation to the $\mathrm{CuO}$ electronic properties which would be consistent with the quite low adsorption energies per water molecule that we have computed. Fig. 7(a) shows the calculated spin polarised density of states (DOS) of pristine $\mathrm{CuO}(111)$, which reveal the

Table 3 Atomic bond distances upon water adsorption or dissociation at the CuO(111) surface, calculated at 1/24,1/8,1/4, 1/3 and 1/2 water coverage for different adsorption configurations. Here, $\mathrm{Cu}_{\text {surf1 }}, \mathrm{Cu}_{\text {surf2, }}, \mathrm{O}_{\mathrm{H}_{2}} \mathrm{O}$ and $\mathrm{H}_{\text {diss }}$ are the surface copper sites, the oxygen atom in the water molecule and the hydrogen radical from the dissociated water, respectively

\begin{tabular}{|c|c|c|c|c|c|}
\hline Water coverage (ML) & $\mathrm{Cu}_{\text {surf } 1}-\mathrm{O}_{\mathrm{H}_{2} \mathrm{O}}$ & $\mathrm{Cu}_{\text {surf } 2}-\mathrm{O}_{\mathrm{H}_{2} \mathrm{O}}$ & $\mathrm{Cu}-\mathrm{OH}$ & $\mathrm{H}_{\mathrm{diss}}-\mathrm{OH}$ & $\mathrm{H}-\mathrm{O}_{\mathrm{H}_{2} \mathrm{O}}$ \\
\hline 1/24 molecular Fig. 2(a) & 2.11 & & & & 0.97 \\
\hline 1/24 molecular Fig. 2(b) & & 2.70 & & & 0.97 \\
\hline 1/8 molecular Fig. $3(a)$ & 2.12 & 2.64 & & & $0.96 / 0.98$ \\
\hline 1/8 molecular Fig. 3(b) & $2.12 / 2.05$ & 2.69 & & & $0.96 / 0.98$ \\
\hline 1/8 molecular Fig. 3(c) & $2.12 / 2.04$ & 2.69 & & & $0.99 / 0.96$ \\
\hline 1/4 molecular Fig. 4(b) & $2.00 / 2.07$ & $2.90 / 3.06$ & & & $0.96 / 1.00$ \\
\hline 1/4 molecular Fig. 4(c) & $2.07 / 2.09$ & & & & $0.96 / 1.00$ \\
\hline 1/4 molecular Fig. 4(d) & $2.00 / 2.07$ & $2.90 / 3.06$ & & & $0.96 / 1.00$ \\
\hline 1/4 dissociative Fig. $4(\mathrm{e})$ & $2.07 / 2.08$ & & 1.87 & 1.73 & $0.96 / 1.00$ \\
\hline 1/3 molecular Fig. 5 (a) & $2.00 / 2.07$ & $2.85 / 3.06$ & & & $0.96 / 1.02$ \\
\hline 1/2 molecular Fig. 5(b) & $2.01 / 2.08$ & $2.91 / 3.11$ & & & $0.96 / 1.02$ \\
\hline
\end{tabular}



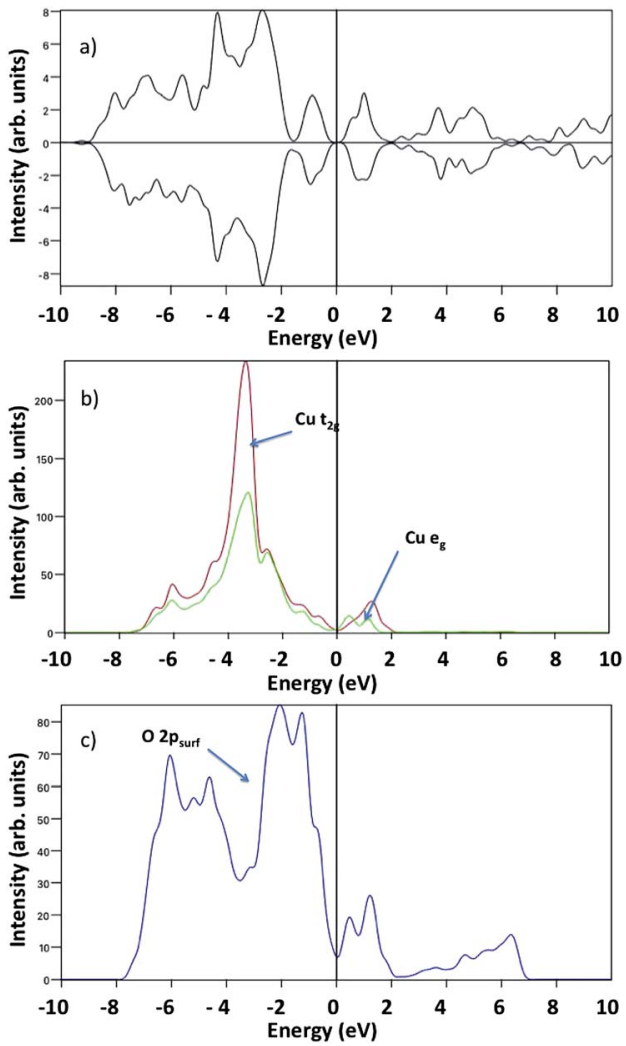

Fig. 7 (a) Total density of states of the pristine $\mathrm{CuO}$ (111) surface. (b) and (c) represent respectively the projected density of states of pristine $\mathrm{CuO}(111)$ of surface $\mathrm{Cu}-\mathrm{t}_{2 \mathrm{~g}}$ and $\mathrm{Cu}-\mathrm{e}_{\mathrm{g}}$ (red and green lines), and surface $0-2 p$ (blue line).

antiferromagnetic nature of the crystal and its semiconducting character, as indicated by the quasi symmetric spin-up and spin-down channels, and the band gap around the Fermi level. The projected density of states (pDOS) shows that, in stoichiometric $\mathrm{CuO}, \mathrm{Cu}-3 \mathrm{~d}$ states consist of two relatively sharp peaks of Cu- $\mathrm{t}_{2 \mathrm{~g}}$ states (red line in Fig. $7(\mathrm{~b})$ ) at $\sim-4 \mathrm{eV}$ and $\sim 1 \mathrm{eV}$, and into two non degenerate $e_{g}$ peaks (green line in Fig. 7(b)) at similar energies, due to the "planar-like" fourfold coordination of $\mathrm{Cu}$ atoms in this crystal. The position of Cu-3d peaks (Fig. 7(b)) and the surface O-2p peak at $\sim-2 \mathrm{eV}$ (Fig. $7(\mathrm{c})$ ) below the Valence Band Maximum (VBM) suggests that $\mathrm{CuO}$ behaves as a charge transfer insulator, in line with other results in the literature. ${ }^{32}$

Fig. 8 shows the surface $\mathrm{Cu}-3 \mathrm{~d}$ and $\mathrm{O}-2 \mathrm{p}$, water $\mathrm{O}-2 \mathrm{p}$ and $\mathrm{H}^{-}$ $1 \mathrm{~s}$ pDOS at $1 / 2 \mathrm{ML}$ coverage. The surface O-2p states show two peaks with a maximum at about $-4.5 \mathrm{eV}$ and $-2 \mathrm{eV}$ below the VBM, while the water O-2p shows three peaks at $-4 \mathrm{eV},-5 \mathrm{eV}$ and $-9 \mathrm{eV}$, where the first indicates a strong hybridization with surface $\mathrm{Cu}$ atoms. Here, we do not observe any evidence of overlapping peaks between water oxygen and surface oxygen. The $\mathrm{H}-1$ s pDOS show an intense double peak around $-9 \mathrm{eV}$, and a less pronounced broad peak between $-7 \mathrm{eV}$ and $-5 \mathrm{eV}$, where the first indicates a strong hybridization with water $\mathrm{O}-2 \mathrm{p}$, whereas the second indicates a weak interaction with surface O-2p.

To analyse the bonding nature of $\mathrm{Cu}-\mathrm{O}$ and $\mathrm{O}-\mathrm{H}$, we determined the Crystal Orbital Overlap Population (COOP) by
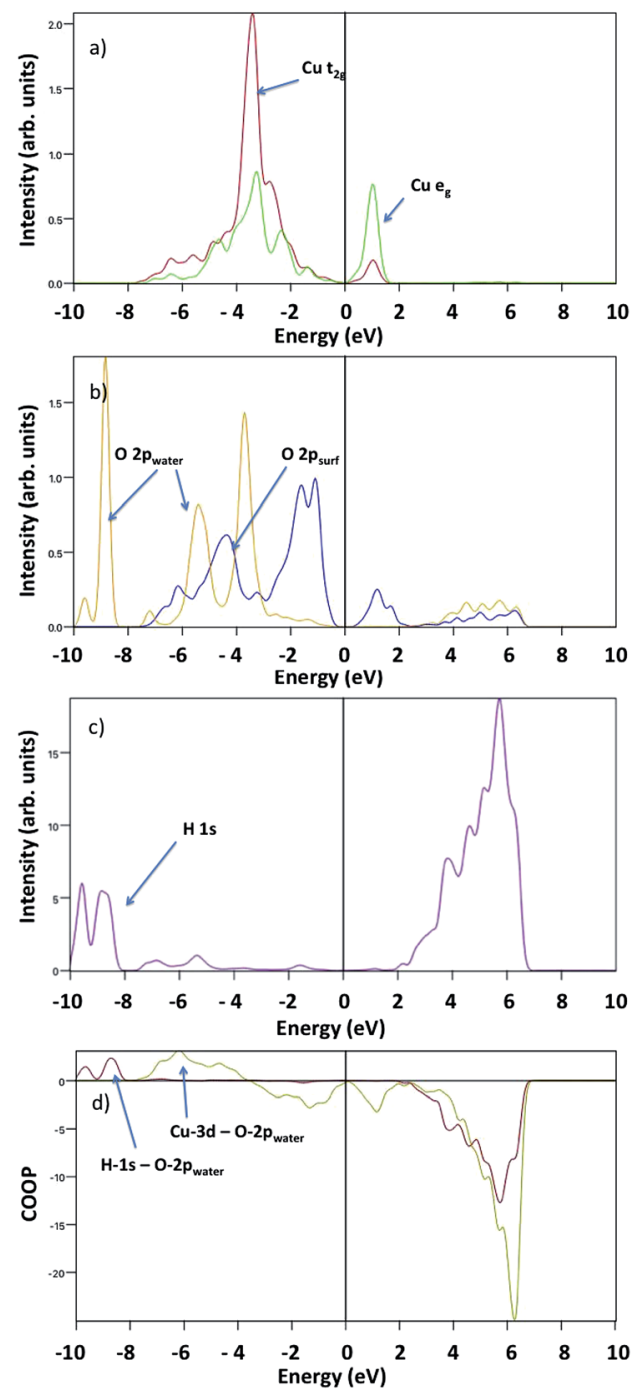

Fig. 8 Projected density of states upon 1/2 ML water adsorption of (a) surface $\mathrm{Cu}-\mathrm{t}_{2 \mathrm{~g}}$ and $\mathrm{Cu}-\mathrm{e}_{\mathrm{g}}$ (red and green lines), (b) surface $\mathrm{O}-2 \mathrm{p}$ (blue line) and water $\mathrm{O}-2 \mathrm{p}$ (yellow line), and (c) water $\mathrm{H}-1$ s (purple line). (d) Calculated crystal orbital overlap population for $\mathrm{H}-1 \mathrm{~s}-\mathrm{O}-2 \mathrm{p}$ (dark red line) and $\mathrm{Cu}-3 d-\mathrm{O}-2 \mathrm{p}$ (dark yellow line).

multiplying the signs of two overlapping atomic wave-functions by the corresponding DOS, providing additional information regarding the bonding character: positive and negative values indicate bonding and antibonding interactions, respectively. Our COOP analysis suggests that the $\mathrm{Cu}-3 \mathrm{~d}-\mathrm{O}-2 \mathrm{p}$ bonding states are between $-4 \mathrm{eV}$ and $-7 \mathrm{eV}$, whereas the $\mathrm{H}-1 \mathrm{~s}-\mathrm{O}-2 \mathrm{p}$ bonding states are between $-10 \mathrm{eV}$ and $-8 \mathrm{eV}$. Other peaks correspond to either non-bonding or anti-bonding states.

No significant qualitative differences are reported at lower water coverages as suggested by the similar pDOS and COOP features in the single water molecule adsorption plot reported in Fig. 9. However, there is a shift of water O-2p peaks together with the $\mathrm{Cu}-3 \mathrm{~d}-\mathrm{O}-2 \mathrm{p}$ bonding peaks towards the Fermi level with relatively low intensity that reflects the lower binding energy at 1/24 ML coverage (see Fig. 9(b) and (d)).

Upon water dissociation, new features appear indicating bond formation, as depicted in Fig. 10. There are two evident 

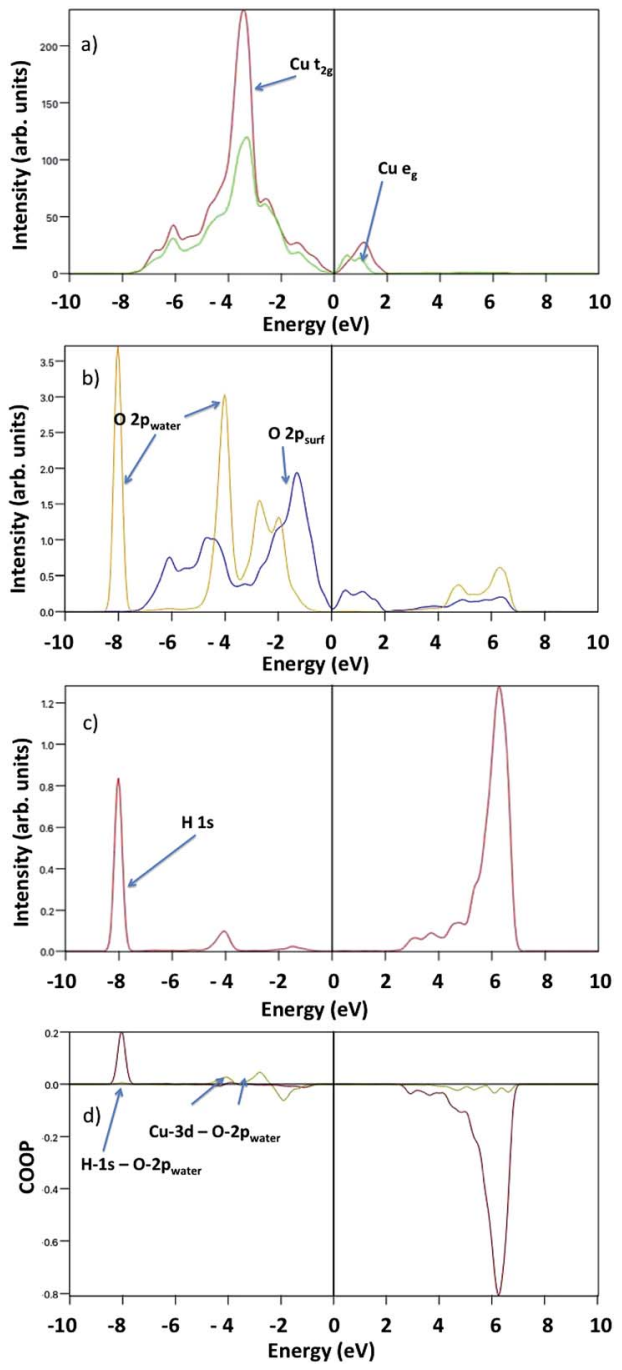

Fig. 9 Projected density of states upon 1/24 ML water adsorption of (a) surface $\mathrm{Cu}-\mathrm{t}_{2 \mathrm{~g}}$ and $\mathrm{Cu}-\mathrm{e}_{\mathrm{g}}$ (red and green lines), (b) surface $\mathrm{O}-2 \mathrm{p}$ (blue line) and water $\mathrm{O}-2 \mathrm{p}$ (yellow line), and (c) water $\mathrm{H}$-1s (red line). (d) Calculated crystal orbital overlap population for $\mathrm{H}-1 \mathrm{~s}-\mathrm{O}-2 \mathrm{p}$ (dark red line) and $\mathrm{Cu}-3 \mathrm{~d}-\mathrm{O}-2 \mathrm{p}$ (dark yellow line).

hybridizations suggested by the overlapping peaks: one at $-8 \mathrm{eV}$ between surface $\mathrm{O}-2 \mathrm{p}$ and atomic $\mathrm{H}-1 \mathrm{~s}$, and another at $-6 \mathrm{eV}$ between $\mathrm{O}-2 \mathrm{p}, \mathrm{H}-1 \mathrm{~s}$ and $\mathrm{Cu}_{\mathrm{tg}}$. COOP analysis confirms the formation of $\mathrm{H}-1$ s bonds with both surface and hydroxyl oxygen, and hydroxyl $\mathrm{O}-2 \mathrm{p}$ bond with $\mathrm{Cu}-3 \mathrm{~d}$.

The same calculations repeated with the Hubbard correction $U=0 \mathrm{eV}$ do not consistently describe a spin configuration consistent with crystal field theory, instead favouring a non magnetic electron configuration. This is because the DFT wrongly describes the partially filled $\mathrm{Cu} 3 \mathrm{~d}$ orbitals, with the d orbital at the Fermi energy. This suggests a metallic character as shown in Fig. 11. Harris et al. correlated the D-HOMO occupation and its position with respect to the Fermi level, the metal chalcogenide bond co-valence and the transition metal chalcogenide bond strength with the catalytic activity. ${ }^{33}$ The more electrons in a D-HOMO, at comparable bond strength, the better the catalytic activity. Smith $e t$ al. performed a DFT calculation
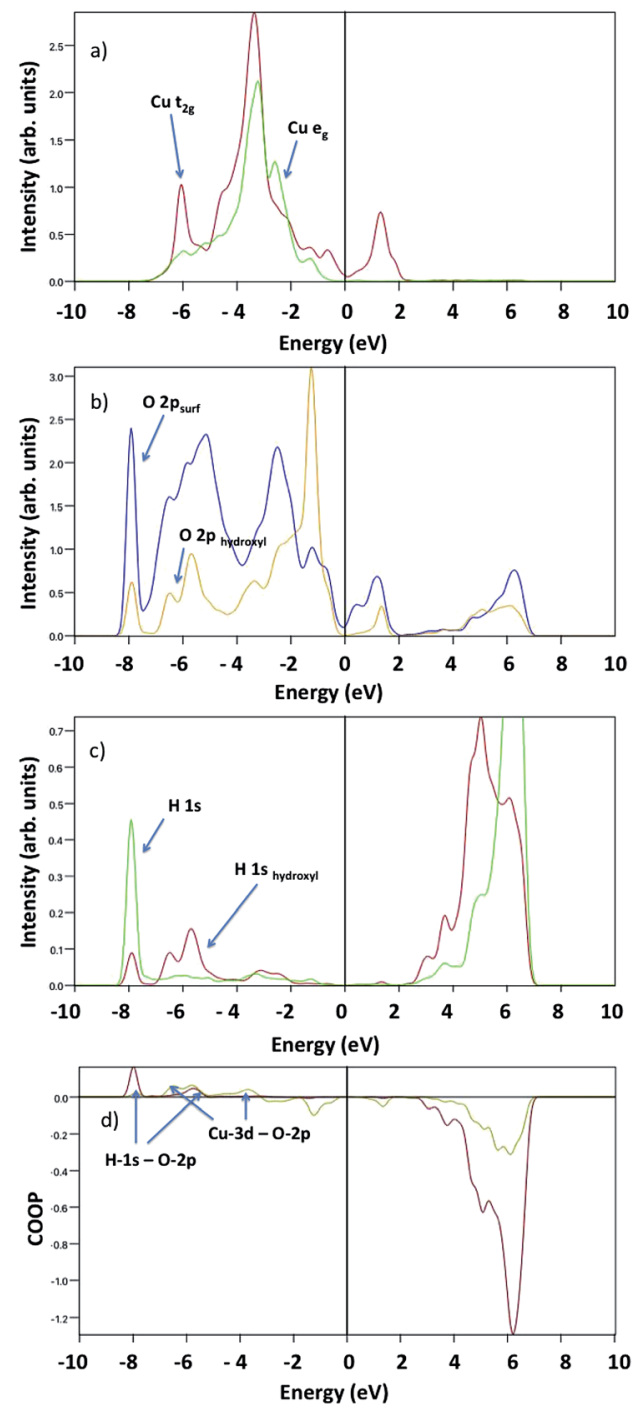

Fig. 10 Projected density of states upon dissociative water adsorption at 1/24 ML coverage of (a) $\mathrm{Cu}-\mathrm{t}_{2 \mathrm{~g}}$ and $\mathrm{Cu}-\mathrm{e}_{\mathrm{g}}$ (red and green line, respectively), (b) water and surface $\mathrm{O}-2 \mathrm{p}$ (yellow and blue line, respectively), and (c) hydroxyl and atomic $\mathrm{H}-1$ s (red and green line). (d) Calculated crystal orbital overlap population for $\mathrm{H}-1 \mathrm{~s}-\mathrm{O}-2 \mathrm{p}$ (dark red line) and $\mathrm{Cu}-3 d-\mathrm{O}-2 \mathrm{p}$ (dark yellow line).

on octahedral transition metal chalcogenide clusters and suggest that the strength of interaction between the metal 3d and the adsorbed $\mathrm{p}$ electrons, together with the $\mathrm{d}$ orbital

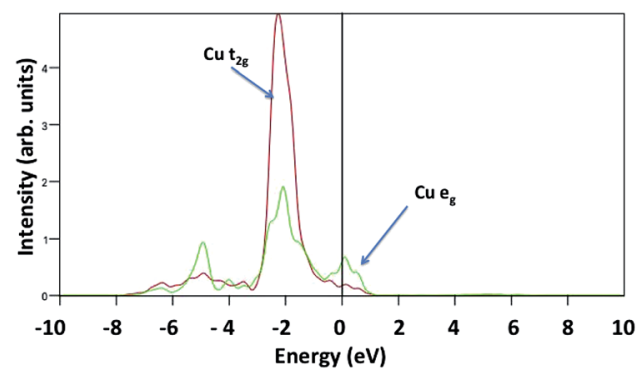

Fig. 11 Projected density of states of the pristine $\mathrm{CuO}(111)$ surface calculated with $U=0$. Here, $\mathrm{Cu}-\mathrm{t}_{2 \mathrm{~g}}$ and $\mathrm{Cu}-\mathrm{e}_{\mathrm{g}}$ are plotted in red and green lines, respectively. 
occupation at the Fermi level, is responsible for the catalytic activity trends. ${ }^{34}$ These considerations suggest that both the occupation of the $d$ states at the Fermi level and the $d-d$ peak energies are crucial to correctly evaluate adsorption energies, which implies that for this crystal the inclusion of the Hubbard correction not only gives a correct description of the magnetic and semiconducting character, but also gives a better description of adsorption energies. This is the origin of the difference between our computed $\mathrm{H}_{2} \mathrm{O}$ adsorption energies and those of ref. 20.

\subsection{Interaction of water with oxygen vacancy sites in $\mathrm{CuO}(111)$}

We now analyse the interaction of water at the $\mathrm{CuO}(111)$ surface in the presence of oxygen vacancies. Oxygen vacancies typically act as catalytically active sites. For bare $\mathrm{CuO}(111)$, we have computed the oxygen vacancy formation energy as follows:

$$
E=E_{\text {surf }+\mathrm{v}_{\mathrm{O}}}+\frac{1}{2} E_{\mathrm{O}_{2}}-E_{\text {surf }},
$$

where $E_{\mathrm{surf}+\mathrm{v}_{\mathrm{O}}}, E_{\mathrm{surf}}$ and $E_{\mathrm{O}_{2}}$ are the energies of the slab after the introduction of a vacancy, the energy of the stoichiometric slab and the energy of a gas phase oxygen molecule, respectively. We investigated vacancy formation at three different water coverages, namely $1 / 24,1 / 8$ and $1 / 2 \mathrm{ML}$, for which the atomic structures are shown in Fig. 12, and the water adsorption energies at the defective $\mathrm{CuO}(111)$ surface and the $\mathrm{O}$ vacancy formation energies after water adsorption are reported in Table 4.

We obtain an energy cost of $1.50 \mathrm{eV}$ for the formation of an oxygen vacancy in the bare $\mathrm{CuO}(111)$ surface, indicating that oxygen atoms will be stable at the surface, consistent with the temperatures required for vacancy formation under vacuum. ${ }^{21}$

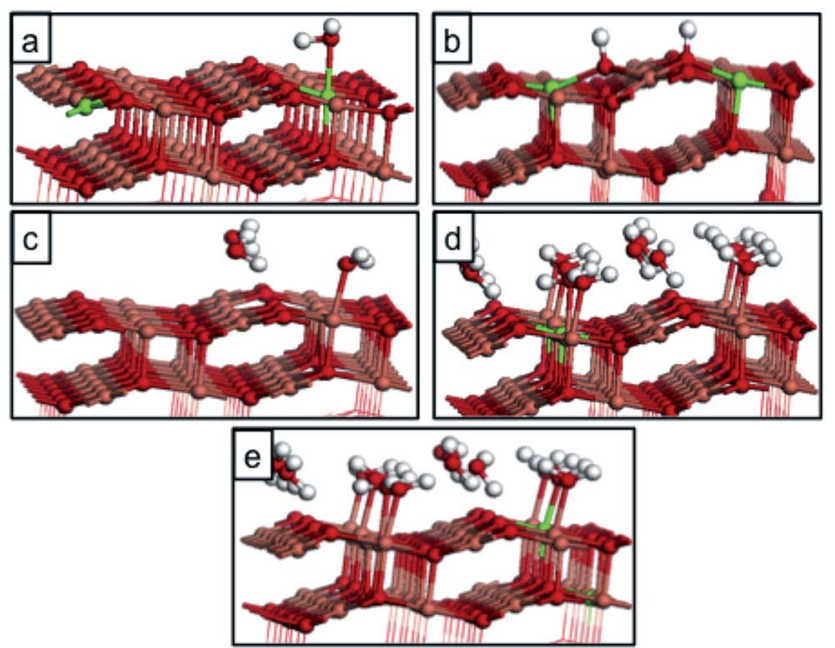

Fig. 12 Atomic structure for water adsorbed on $\mathrm{CuO}(111)$ with a surface oxygen vacancy. (a) molecular water at $1 / 24 \mathrm{ML}$ coverage, (b) dissociative water adsorption at $1 / 24 \mathrm{ML}$ coverage, (c) molecular water adsorption at $1 / 8 \mathrm{ML}$ coverage, ( $d$ and e) water adsorption structures at different $O$ vacancy sites at $1 / 2 \mathrm{ML}$ coverage. Reduced $\mathrm{Cu}^{+}$species resulting from the formation of the oxygen vacancy are shown by green spheres.
Table 4 Adsorption energies of water (per molecule) at the defective $\mathrm{CuO}(111)$ surface with one $\mathrm{O}$ vacancy, and vacancy formation energy at water coverages of $1 / 24,1 / 8$ and $1 / 2 \mathrm{ML}$. Energies are referenced to gas phase water and the bare $\mathrm{CuO}(111)$ surface

\begin{tabular}{lll}
\hline Water coverage & Ads. $E(\mathrm{eV})$ & $V_{\mathrm{O}} E(\mathrm{eV})$ \\
\hline 1/24 ML molecular (Fig. 12(a)) & -0.08 & 1.75 \\
1/24 ML dissociative (Fig. 12(b)) & -0.73 & 1.08 \\
1/8 ML molecular (Fig. 12(c)) & -0.30 & 2.48 \\
1/2 ML molecular (Fig. 12(d)) & -0.58 & 1.81
\end{tabular}

However, Maimaiti et al. show that $\mathrm{CuO}$ is easily reduced by hydrogen and it is therefore important to investigate the interaction of water with the defective $\mathrm{CuO}$ surface. The computed adsorption energies in Table 4 show that when an $\mathrm{O}$ vacancy is present on the surface, the water adsorption energies depend on the coverage, with the molecular adsorption at 1/24 ML being the least favourable. At this coverage, dissociative water adsorption is more stable by almost $0.7 \mathrm{eV}$. At higher coverages molecular water adsorption becomes more stable, and shows similar energetics to water adsorption at the oxidised $\mathrm{CuO}$ surface. If we consider $\mathrm{O}$ vacancy formation, with water already adsorbed on the oxidised $\mathrm{CuO}$ surface at the same coverages, there is a notable coverage dependence in the vacancy formation energies. For example, the least favourable vacancy is at $1 / 8$ ML coverage and the $1 / 2 \mathrm{ML}$ water coverage also has a higher vacancy formation energy than the clean surface. Thus, the presence of water may act to increase the temperature at which oxygen vacancies form in $\mathrm{CuO}$ (111).

From this first description of the interaction of water with $\mathrm{CuO}(111)$ at zero temperature and pressure, we conclude that there is some dependence of water stability on water coverage. There is a gain in energy upon dissociation on both stoichiometric and reduced $\mathrm{CuO}(111)$ surfaces. Therefore, when water adsorbs on a stoichiometric surface it can likely form a mix of molecular and dissociated water. We can conclude that such analysis, within bare DFT calculations at zero temperature and zero pressure conditions predicts the surface to have a mixture of molecular water, $\mathrm{OH}$ and $\mathrm{H}$ terminations; the precise details of which will depend on the state of the surface.

\section{Thermodynamics of water adsorption on $\mathrm{CuO}(111)$}

To analyse the thermodynamic stability of an adsorbate-surface system, such as water on a metal oxide when exposed to a specific environment, it is crucial to include the effects of temperature and pressure of the surrounding atmosphere. This is achieved by employing ab initio atomistic thermodynamics. ${ }^{37}$ Within this approach the system is considered to be in thermodynamic equilibrium with a gas reservoir, representing the atmosphere, that exchanges particles with the system without changing its chemical potential. The surface energy can be calculated as the change in Gibbs free energy of the surface due 
to the formation of a particular composition, which, normalized by the unit area, is:

$$
\gamma\left(p_{\mathrm{i}}, T\right)=\frac{1}{A}\left[G-\sum_{\mathrm{i}} N_{\mathrm{i}} \mu_{\mathrm{i}}\left(p_{\mathrm{i}}, T\right)\right],
$$

here $G$ is the Gibbs free energy of the slab with surface area $A, \mu_{i}$ $\left(p_{i}, T\right)$ is the chemical potential of the species i present in the system, (i.e. $\mathrm{i}=\mathrm{Cu}, \mathrm{O}$ or $\mathrm{H}$ ), and $N_{\mathrm{i}}$ is the total number of atoms in the component $i$ of the system. To consider the stability of the adsorbate on the surface, we compute the free energy of water binding at $\mathrm{CuO}(111)$, which is the difference between the clean and adsorbate-surface systems:

$$
\gamma_{\text {bond }}\left(p_{\mathrm{i}}, T\right)=\gamma_{\text {clean }}\left(p_{\mathrm{i}}, T\right)-\gamma_{\text {surf-mol }}\left(p_{\mathrm{i}}, T\right),
$$

where $\gamma_{\text {clean }}$ is the free energy of the bare surface and $\gamma_{\text {surf-mol }}$ $\left(p_{\mathrm{i}}, T\right)$ is the energy of the adsorbate-surface system. Here $\gamma_{\mathrm{bond}}$ $\left(p_{i}, T\right)$ represents the magnitude of the surface- $\mathrm{H}_{2} \mathrm{O}$ interaction as a function of the chemical potential of the species in the atmosphere. Then, in order to explicitly write the dependence of the energy on $p$ and $T$, we can express the chemical potential of oxygen and water as follows:

$$
\begin{aligned}
& \mu(p, T)=\frac{1}{2}\left[E_{\mathrm{O}_{2}}+\mu_{\mathrm{O}_{2}}(p, T) k_{\mathrm{B}} T \ln \left(\frac{p_{\mathrm{O}_{2}}}{p^{0}}\right)\right], \\
& \mu(p, T)=\left[E_{\mathrm{H}_{2} \mathrm{O}}+\mu_{\mathrm{H}_{2} \mathrm{O}}(p, T) k_{\mathrm{B}} T \ln \left(\frac{p_{\mathrm{H}_{2} \mathrm{O}}}{p^{0}}\right)\right],
\end{aligned}
$$

Each chemical potential can be expressed as a function of the thermodynamic variables $T$ and $p$. Here, we explicitly give the expression for water and oxygen, since our analysis includes these as independent variables. We can now, for convenience, re-define these quantities so that $0 \mathrm{eV}$ represents the oxygen and water rich limiting conditions, and the plot is constructed as a function of $\Delta \mu_{\mathrm{O}}$ and $\Delta \mu_{\mathrm{H}_{2} \mathrm{O}}$. The rich and poor limit for the chemical potential can be calculated considering oxygen and water to be in thermal equilibrium with the $\mathrm{CuO}(111)$ surface. The oxygen-poor limit corresponds to the condition when $\mathrm{CuO}$ dissociates into $\mathrm{Cu}$ and $\mathrm{O}_{2}$, while the oxygen-rich limit corresponds to the condition in which oxygen molecules condense on the surface. Analogous considerations apply for water, where an additional restriction has to be applied for the water-rich limit by considering water staying in the gas phase at any temperature or pressure. For this specific system, the limits for the chemical potentials are $0 \geq \Delta \mu_{\mathrm{O}} \geq-3.0 \mathrm{eV}$ and $0 \geq \Delta \mu_{\mathrm{H}_{2} \mathrm{O}} \geq$ $-0.91 \mathrm{eV}$. A more detailed description of the methodology used here, as well as the derivation of these quantities, can be found in previous works, where a similar approach has been used to investigate comparable systems. ${ }^{19,24,37,38}$

By employing this methodology, we are able to calculate the binding energy of the water-surface system at different water coverages. This will result in a two dimensional surface, plotted in a three dimensional space, where the $x-y$ plane is here $\Delta \mu_{\mathrm{O}^{-}}$ $\Delta \mu_{\mathrm{H}_{2} \mathrm{O}}$, and the $z$ axis is the binding energy. In the plot we consider the binding of the water in equilibrium with the environment. This means that the oxygen and the water in the atmosphere will have an effect on the binding energy of the molecule on the surface and the CuO bulk is not affected by any change in the chemical potentials. Therefore, we are able to plot the stability of the adsorbate-surface system and calculate the desorption pressure and temperature at different water coverages. If we then project the lowest binding energy surface $\gamma_{\text {bond }}$ on the $x-y$ plane, we obtain the phase diagram shown in Fig. 13. We use eqn (5) and (6) to express the chemical potential of oxygen and water in terms of partial pressure at different temperatures. The temperature range of interest is between 300 $\mathrm{K}$ and $1200 \mathrm{~K}$, while the typical experimental pressure range is between $1 \mathrm{~atm}$ and $10^{-14} \mathrm{~atm}$ (UHV). Fig. 13 shows the water adsorption phase diagram, which is clearly divided in six different areas, and each area represents, for a given value of the chemical potential of the species, a thermodynamically stable structure. For a given temperature, at high values of the partial pressures of each species, the most stable structure is $\mathrm{CuO}(111)$ with a high water coverage. If we then move to lower partial pressure in each direction, the binding of water at lower coverage becomes more favourable, showing a relatively narrow area where an intermediate coverage, from 8 to 1 water molecules per super-cell (corresponding to $1 / 3$ to $1 / 24 \mathrm{ML}$ ), is stable. Finally at a very low partial pressure of each species, a clean $\mathrm{CuO}(111)$ surface is stable. We can immediately see that both water and oxygen chemical potentials play the same role in the water adsorption-desorption trend. However, this is only a qualitative observation, since the actual transitions occur at different conditions if we consider the two directions of $\Delta \mu_{\mathrm{O}}$ and $\Delta \mu_{\mathrm{H}_{2} \mathrm{O}}$.

For quantitative consideration, we can group the above mentioned areas into three main groups, namely the red area, which represents the stability region of the clean surface, the black area, which represents the stability region of high

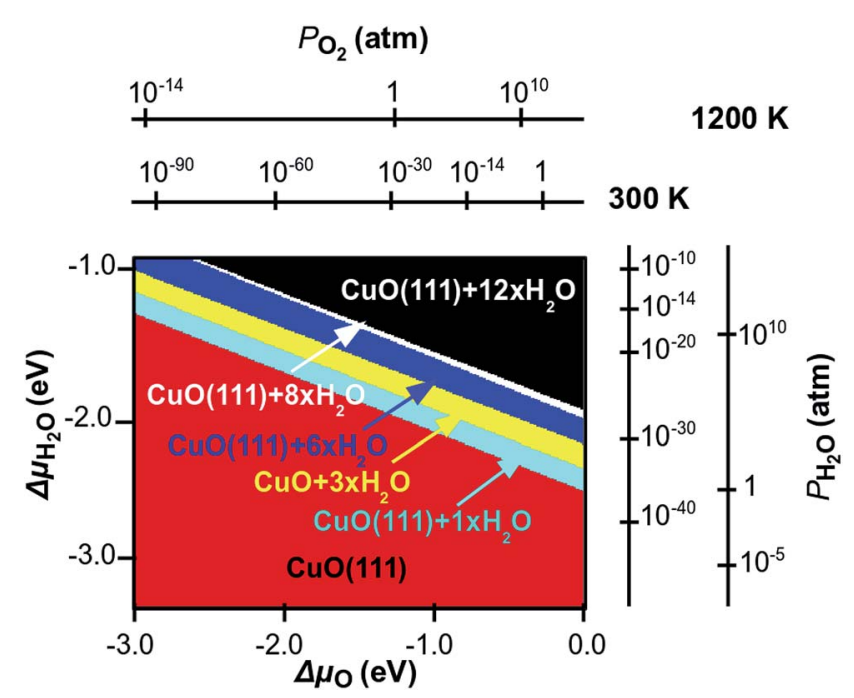

Fig. 13 Surface phase diagram of stable structures of $\mathrm{CuO}(111)$ in equilibrium with a "humid environment", as a function of $\Delta \mu_{\circ}$ and $\Delta \mu_{\mathrm{H}_{2} \mathrm{O}}$ in the gas phase. The additional axes show the corresponding pressure scales at $T=300 \mathrm{~K}$ and $1200 \mathrm{~K}$. Coloured areas are associated to different water coverages as follows: black $(1 / 2 \mathrm{ML})$, white $(1 / 3 \mathrm{ML})$, blue (1/4 ML), yellow (1/8 ML), turquoise (1/24 ML) and red (pristine). 
coverage and the remaining area, which represents the stability region of medium coverage. If we fix the chemical potential of one species and let the other change, we can identify the conditions for phase-transitions that involve water desorption. If we fix the chemical potential of oxygen to be $0 \mathrm{eV}$ and let $\Delta \mu_{\mathrm{H}_{2} \mathrm{O}}$ change, we are simulating an oxygen rich atmosphere at different humidity. Within these conditions if water pressure is at $1 \mathrm{~atm}$, we find that the transition between high coverage and medium coverage is at $\sim 800 \mathrm{~K}$, while the transition between medium coverage and a clean surface after desorption is at $\sim 1000 \mathrm{~K}$, so that $\mathrm{CuO}$ will be covered with water up to high temperatures. This would be relevant for using $\mathrm{CuO}$ in catalysis where surface water could be involved in chemical reactions. The values of the transition temperature drop to $\sim 400 \mathrm{~K}$ and $\sim 650 \mathrm{~K}$ when the partial pressure of the water is $10^{-14} \mathrm{~atm}$. If we repeat this analysis, but this time fixing the chemical potential of water at rich conditions and let the oxygen chemical potential vary, we calculate the transition between high and medium coverage to occur at very high temperatures above $3000 \mathrm{~K}$, while if the pressure of the oxygen drops to $10^{-14}$ (UHV), the same transition will occur at $\sim 950 \mathrm{~K}$. In this case the other transition would occur outside of our scale, where the $\mathrm{CuO}$ bulk dissociates into $\mathrm{Cu}$ and $\mathrm{O}$, therefore we do not consider this here. The effect of the partial pressure of oxygen and water on the water desorption process is the same, however it takes place at different temperatures in each case.

It is interesting to analyse the same transition when the temperature of the environment is fixed at $450 \mathrm{~K}$, which is a typical condition for catalytic reactions. If the oxygen partial pressure is $1 \mathrm{~atm}$., then the transition from high to medium coverage occurs when water partial pressure is below $10^{-14} \mathrm{~atm}$. The transition from medium coverage of water to a bare surface is at $10^{-10} \mathrm{~atm}$. On the other hand if we fix $p_{\mathrm{O}_{2}}$ around $10^{-14}$ (UHV) then the same transitions occur at $p_{\mathrm{H}_{2} \mathrm{O}}=10^{-5}$ and $10^{-14}$ atm.

The phase diagram represented here is calculated for adsorption energies computed for a Hubbard term $U=4.0 \mathrm{eV}$. The effect on the water binding energies is relatively small for a change of $U$ up to $7.0 \mathrm{eV}$ and it would not bring any substantial qualitative change in the extrapolated adsorption-desorption. However what is most important is the effect of including the $+U$ correction in the first place (with a sufficiently large value of $U$ ) failure to account for the correlated nature of the $\mathrm{Cu}^{2+}$ oxidation state in the thermodynamics analysis will lead to very different results, given the large difference in water binding energy between our calculations and those of Zhang et al. ${ }^{20}$ After these considerations we can conclude that water dissociation may occur only in the areas of the phase diagram where water adsorbed on the surface is stable. As seen in the last section, this would bring a gain in energy, which therefore indicates that the process is thermodynamically favourable. A further energy gain will occur after water dissociation at the vacancy site, with the formation of $\mathrm{H}$ species adsorbed at the $\mathrm{O}$ sites, and the oxygen filling the vacancy. However, although this process is favoured, it would occur outside our limits, since oxygen vacancies have an energy cost to form, which is confirmed in previous analysis. ${ }^{21}$ From the previous section we predict that water can be present on the $\mathrm{CuO}(111)$ surface both molecularly and dissociated (forming hydroxyl groups on the surface), and the necessary thermodynamic conditions are within the black area. However, after dissociation and hydrogen desorption the environmental conditions to analyse in detail the stability of the surface will change, and a detailed investigation of such conditions is beyond the scope of this work. Also, although in the previous section we concluded that water desorption at the vacancy site is highly favourable, the vacancy formation occurs outside the stability of the $\mathrm{CuO}$ surface. This result indicates the fundamental role of the chemical potential of both water and oxygen in the description of molecule-surface interaction.

\section{Conclusions}

We performed DFT calculations in order to analyse the energetics and the structure of $\mathrm{H}_{2} \mathrm{O}$ adsorption on the stoichiometric and reduced $\mathrm{CuO}(111)$ surface at different water coverages. Our results show that, on the stoichiometric surface, the most stable structure is the one in which water is adsorbed at the $\mathrm{Cu}_{\text {surf2 }}$ site. However, the adsorption energy indicates a very small difference if compared to the adsorption at the $\mathrm{Cu}_{\text {surf1 }}$ site. We found the adsorption energy to increase with the coverage up to $1 / 2 \mathrm{ML}$, while a dissociative adsorption is stable with a very small energy gain at low coverages. We found that water stabilizes a stoichiometric surface, while having little influence on the magnetic moment and charge of the surface copper atoms. We impose the Hubbard correction to the energy to describe electron localization and we find a significant effect on the adsorption energies for values up to $U=4.0 \mathrm{eV}$, while at higher values, up to $U=7.0 \mathrm{eV}$, the effect is negligible. To include realistic environmental variables such as temperature and pressure, we employ our DFT results within the ab initio thermodynamics framework. This approach allows us to calculate the conditions for water desorption, and we found a few interesting transitions. When the pressure is $1 \mathrm{~atm}$, we calculate the transition between high coverage and medium coverage to be at $\sim 800 \mathrm{~K}$, while total water desorption occurs only at $\sim 1000 \mathrm{~K}$. The same values of temperature drop respectively to $\sim 400$ and $\sim 650 \mathrm{~K}$ when the partial pressure of the water is $10^{-14} \mathrm{~atm}$. It is crucial to point out the fundamental role of the $a b$ initio thermodynamics as a methodology to predict surface reactivity. In fact, while plain DFT calculations, which consider the system to be at $T=0$ and $p=0$, predict an $\mathrm{H}$ and $\mathrm{OH}$ terminated surface, after the inclusion of a thermodynamic variable we conclude that water will not dissociate on $\mathrm{CuO}(111)$ under typical reaction conditions.

\section{Conflicts of interest}

There are no conflict to declare.

\section{Acknowledgements}

The authors gratefully acknowledge the financial support from the National Natural Science Foundation of China (No. 51323011), and from the Science Foundation Ireland (SFI) 
through the US-Ireland R\&D Partnership Program, grant number SFI 14/US/E2915 and the European Commission through the COST Action CM1104 "Reducible Metal Oxides, Structure and Function”. We acknowledge access to computing resources at Tyndall provided by SFI and by the SFI and Higher Education Authority funded Irish Centre for High End Computing.

\section{References}

1 Z. Fang, D. Wang, P. Chang, W. Tseng and J. Lu, Appl. Phys. Lett., 2004, 85, 5929.

2 C. Li, D. Zhang, X. Liu, S. Han, T. Tang, J. Han and C. Zhou, Appl. Phys. Lett., 2003, 82, 1613.

3 S. C. Ray, Sol. Energy Mater. Sol. Cells, 2001, 68, 307.

4 K. Hardee and A. Bard, J. Electrochem. Soc., 1977, 124, 215.

5 F. Marabelli, G. Parravicini and F. Salghetti-Drioli, Phys. Rev.

B: Condens. Matter Mater. Phys., 1995, 52, 1433.

6 J. Ghijsen, L. H. Tjeng, J. Elp and H. Eskes, Phys. Rev. B: Condens. Matter Mater. Phys., 1988, 38, 11322.

7 M. Heinemann, B. Eifert and C. Heiliger, Phys. Rev. B: Condens. Matter Mater. Phys., 2013, 87, 115111.

8 M. Nolan and S. D. Elliott, Phys. Chem. Chem. Phys., 2006, 8, 5350.

9 K. Terakura, T. Oguchi, A. R. Williams and J. Kubler, Phys. Rev. B: Condens. Matter Mater. Phys., 1984, 30, 4373.

10 M. Grioni, M. T. Czyzyk, F. M. F. de Groot, J. C. Fuggle and B. E. Watts, Phys. Rev. B: Condens. Matter Mater. Phys., 1989, 39, 4886.

11 W. Y. Ching, Y. N. Xu and K. W. Wong, Phys. Rev. B: Condens. Matter Mater. Phys., 1989, 40, 7684.

12 C. E. Ekuma, V. I. Anisimov, J. Moreno and M. Jarrell, Eur. Phys. J. B, 2014, 87, 40949.

13 V. I. Anisimov, J. Zaanen and O. K. Andersen, Phys. Rev. B: Condens. Matter Mater. Phys., 1991, 44, 943.

14 R. C. Albers, N. E. Christensen and A. Svane, J. Phys.: Condens. Matter, 2009, 21, 343201.

15 A. Svane and O. Gunnarsson, Phys. Rev. Lett., 1990, 65, 1148. 16 M. Takahashi and J.-I. Igarashi, Phys. Rev. B: Condens. Matter Mater. Phys., 1997, 56, 12818.
17 M. Fronzi, A. Iwaszuk, A. Lucid and M. Nolan, J. Phys.: Condens. Matter, 2016, 28, 074006.

18 M. Posternak, A. Baldereschi and B. Delley, J. Phys. Chem. C, 2009, 113, 15862.

19 M. Fronzi, S. Piccinin, B. Delley, E. Traversa and C. Stampfl, Phys. Chem. Chem. Phys., 2009, 11, 9188.

20 J. Zhang, R. Zhang, B. Wang and L. Ling, Appl. Surf. Sci., 2016, 364, 758.

21 Y. Maimaiti, M. Nolan and S. Elliott, Phys. Chem. Chem. Phys., 2014, 16, 3036.

22 J. J. Carey and M. Nolan, Appl. Catal., B, 2016, 197, 324.

23 C. Stampfl, Catal. Today, 2005, 105, 17.

24 K. Reuter and M. Scheffler, Phys. Rev. B: Condens. Matter Mater. Phys., 2001, 65, 035406.

25 J. P. Perdew, K. Burke and M. Ernzerhof, Phys. Rev. Lett., 1996, 77, 3865.

26 G. Kresse and J. Furthmuller, Comput. Mater. Sci., 1996, 6, 15.

27 P. E. Blöchl, Phys. Rev. B: Condens. Matter Mater. Phys., 1994, 50, 17953.

28 M. Nolan, S. C. Parker and G. W. Watson, Surf. Sci., 2005, 595, 223.

29 M. Nolan, ACS Appl. Mater. Interfaces, 2012, 4, 5863.

30 S. Grimme, J. Antony, S. Ehrlich and S. Krieg, J. Chem. Phys., 2010, 132, 154104.

31 J. Carrasco, J. Klimeš and A. Michaelides, J. Chem. Phys., 2013, 138, 024708.

32 Y. Wang, S. Lany, J. Ghanbaja, Y. Fagot-Revurat, Y. P. Chen, F. Soldera, D. Horwat, F. Mücklich and J. F. Pierson, Phys. Rev. B: Condens. Matter Mater. Phys., 2016, 94, 245418.

33 S. Harris and R. Chianelli, J. Catal., 1984, 86, 400.

34 T. S. Smith and K. H. Johnson, Catal. Lett., 1994, 28, 361.

35 M. Nolan, A. Iwaszuk and K. A. Gray, J. Phys. Chem. C, 2014, 118, 27890.

36 A. Iwaszuk, A. K. Lucid, K. M. Razeeb and M. Nolan, J. Mater. Chem. A, 2014, 2, 18796.

37 K. Reuter and M. Scheffler, Phys. Rev. B: Condens. Matter Mater. Phys., 2003, 68, 045407.

38 M. Fronzi, S. Piccinin, B. Delley, E. Traversa and C. Stampfl, RSC Adv., 2014, 4, 12245. 